Paper 45
$\begin{aligned} & \text { OCivil-Comp Press, 2008 } \\ & \text { Proceedings of the Ninth International Conference } \\ & \text { on Computational Structures Technology, } \\ & \text { B.H.V. Topping and M. Papadrakakis, (Editors), } \\ & \text { Civil-Comp Press, Stirlingshire, Scotland }\end{aligned}$

\title{
A Wave Based Prediction Technique for the Dynamic Response Analysis of Plates with Random Point Mass Distributions
}

\author{
K. Vergote, B. Van Genechten, B. Pluymers, D. Vandepitte and W. Desmet \\ Department of Mechanical Engineering \\ K.U.Leuven, Belgium
}

\begin{abstract}
The Finite Element Method and the Statistical Energy Analysis are most commonly used for the analysis of the dynamical behaviour of mechanical structures. However, the first is limited to applications in the low-frequency range, while the latter is only applicable high frequencies. In the mid-frequency range there is a lack of methods techniques to model assemblies with the combination of components with a lowfrequency behaviour and components with a high-frequency behaviour. This paper presents a deterministic Wave Based Method for efficient Monte Carlo simulations of the dynamic response of flexible plates with point masses distributed randomly. The effect of random point mass distributions on the dynamic response can be representative for the typical variability encountered in mechanical systems. The performance of the proposed method is illustrated through a numerical validation example.
\end{abstract}

Keywords: structural dynamics, mid-frequency, wave based method, deterministic, probabilistic, Monte Carlo simulation.

\section{Introduction}

Due to the ever more restrictive legal regulations and the increased customer comfort expectations, the vibrational and acoustical behaviour of a product has become an important criterion. Over the past decades, numerical simulation techniques have settled as a vital part of the design process. They have shortened the design cycle and reduced the number of (expensive) physical prototypes significantly. Since the vibroacoustic behaviour of a product is often predominantly determined by the steady-state dynamic deformations of the involved mechanical structures, this paper focusses on the numerical prediction of those deformations. 
Currently, there are two groups of numerical prediction techniques, i.e. the deterministic and the probabilistic. From the group of deterministic prediction techniques, the Finite Element Method (FEM) [1] is most commonly used. The FEM discretises the problem domain into large number of small elements in which the dynamic field variables are approximated by simple polynomial shape functions. As the spatial variation of the dynamic response increases with frequency, the number of elements needed to accurately describe the dynamic response increases. This results in an exponentially increasing computational load when frequency increases, limiting the practical application of the FEM to problems in the low-frequency range. A second problem prohibiting the use of FEM, and other deterministic techniques, at higher frequencies, is that the response of a system becomes increasingly sensitive to small perturbations of its properties when frequency increases. Therefore variabilities have to be taken into account at higher frequencies.

Another well-known deterministic prediction technique is the Boundary Element Method (BEM) [2, 3]. It is based on a boundary integral formulation of the problem. As a result, only the boundary of the considered problem domain has to be descretised. Within the boundary elements, the dynamic field variables are approximated by simple polynomial shape functions. Similar to the FEM, the BEM is limited to low-frequency applications.

Among the probabilistic techniques, the methods based on Statistical Energy Analysis (SEA) [4] are mostly used. SEA methods divide the system in a limited number of subsystems, for which frequency- and space-averaged energy levels are predicted. These methods are much less computationally expensive, but are only applicable if all subsystems exhibit, amongst others, a high modal overlap, which limits their use to high-frequency applications.

Next to the SEA, a commonly used implementation of probabilistic numerical analysis is the Monte Carlo simulation [12,13]. This technique requires an (often prohibitively) large number of deterministic calculations which limits its applicability to low-frequency problems.

For mid-frequency applications, currently no efficient and mature prediction techniques exist. Recently a new deterministic technique, called the Wave Based Method (WBM), has been developed [5], which has shown to be able to successfully tackle structural dynamic problems in the mid-frequency range. The method is based on an indirect Trefftz approach [6] in that the dynamic response variables are described in terms of wave functions which exactly satisfy the governing differential equations. In that way, no approximation error is made inside the problem domain. However, the wave functions may violate the boundary and continuity conditions. Therefore, the approximation errors on the domain boundaries and interfaces are minimized by means of a weighted residual formulation. This approach leads to substantially smaller system matrices as compared to the FEM, which results in an enhanced computational efficiency. As a result, the WBM is able to not only tackle applications in the lowfrequency range, but also in the mid-frequency range.

However, when the frequency increases many structures consist of some subsys- 
tems with a probabilistic behaviour and some subsystems with a deterministic behaviour. In this prospect it is useful to develop a deterministic methodology which can handle both the deterministic and the probabilistic behaviour. Langley [11] has shown that when there is enough uncertainty in the dynamic properties of a system, the response statistics become insensitive to the way in which the system is randomised. Therefore, this paper proposes an approach based on the deterministic WBM for structural dynamics $[7,8,9,10]$ which is able to perform Monte Carlo simulations with point masses at random locations efficiently. These random point mass distributions introduce the desired variability of the population.

The paper starts with the basic concepts of the WBM for the analysis of plate bending problems. The following section describes how point masses are introduced in the WBM formulation. Thereafter, the theory is expanded to the use of several point masses and an efficient implementation is proposed. A numerical example demonstrates the validity and the efficiency of the presented method.

\section{Basic concepts of the WBM for plate bending}

This section describes briefly the basic principles of the WBM for the analysis of plate bending problems [7]. As mentioned in the introduction, the WBM belongs to the category of indirect Trefftz methods [6]. The WBM partitions the problem domain into a small number of subdomains. According to the Trefftz principle, the field variables within each subdomain are approximated by an expansion of basis functions, which exactly satisfy the governing dynamic equations. In this way, there is only an approximation error introduced in the boundary conditions and in the conformity between the subdomains. These errors are forced to zero in an integral sense through the application of a Galerkin-like weighted residual formulation.

\subsection{Problem definition}

Two important plate bending theories exist, namely the Kirchhoff theory [14] and the Reissner-Mindlin theory $[15,16]$. The WBM, described in this paper, is based on the Kirchhoff theory. For thin plates, it is shown that the Kirchhoff theory yields accurate results $[17,18]$.

According to the Kirchhoff theory, the steady-state out-of-plane displacements $w_{z}$ are governed by the following fourth-order partial differential equation,

$$
\nabla^{4} w_{z}(x, y)-k_{b}^{4} w_{z}(x, y)=\frac{F_{z}}{D} \delta\left(x_{F}, y_{F}\right)
$$

where $\nabla^{4}=\frac{\partial^{4}}{\partial x^{4}}+2 \frac{\partial^{4}}{\partial x^{2} \partial y^{2}}+\frac{\partial^{4}}{\partial y^{4}}$. The plate bending wave number $k_{b}$ and plate 
bending stiffness $D$ are defined as,

$$
\begin{array}{r}
k_{b}=\sqrt[4]{\frac{\rho h \omega^{2}}{D}}, \\
D=\frac{E(1+j \eta) h^{3}}{12\left(1-\nu^{2}\right)},
\end{array}
$$

with $h, E, \nu, \eta$ and $\rho$, respectively, the plate thickness, the elasticity modulus, the Poisson coefficient, the material loss factor and the plate material density, and $j=\sqrt{-1}$. The plate is excited with a normal point force $F$ in point $\left(x_{F}, y_{F}\right)$.

Since the Kirchhoff theory is governed by a fourth-order partial differential equation, two boundary conditions need to be imposed at the plate boundary $\Gamma$. In case of clamped edges, the out-of-plane displacement and the normal rotation are prescribed,

$$
\left\{\begin{array}{l}
R_{w_{z}}=w_{z}-\bar{w}_{z}=0 \\
R_{\theta_{n}}=\mathcal{L}_{\theta_{n}}\left[w_{z}\right]-\bar{\theta}_{n}=0
\end{array}\right.
$$

with $\bar{w}_{z}$ and $\bar{\theta}_{n}$ the prescribed values for the transverse displacement and the normal rotation, here both zero, and $\mathcal{L}_{\theta_{n}}$ the differential operator for the normal rotation, defined as:

$$
\mathcal{L}_{\theta_{n}}=-\frac{\partial}{\partial n}
$$

with $n$ the in-plane normal direction of the plate boundary $\Gamma$ of the domain. The reader is referred to [7] for the boundary conditions for simply supported and free edges.

\subsection{Field variable expansion}

The steady-state out-of-plane displacement $w_{z}$ is approximated by the following field variable expansion,

$$
w_{z}(\mathbf{x}) \approx \hat{w}_{z}(\mathbf{x})=\sum_{b=1}^{n_{b}} w_{b} \Psi_{b}(\mathbf{x})+\hat{w}_{F}(\mathbf{x})=\mathbf{\Psi}(\mathbf{x}) \cdot \mathbf{w}+\hat{w}_{F}(\mathbf{x}),
$$

with $\Psi_{b}(\mathbf{x})$ and $w_{b}$, respectively, the wave functions and the corresponding contribution factors, and $\Psi(\mathrm{x})$ and $\mathrm{w}$ vectors containing those wave functions and contribution factors. The particular solution $\hat{w}_{F}(\mathbf{x})$ satisfies the inhomogeneous part of equation (1), arising from an external loading. The displacement field of an infinite plate excited with a normal point force [19] is selected as particular solution,

$$
\hat{w}_{F}=-\frac{j F}{8 k_{b}^{2} D}\left[H_{0}^{(2)}\left(k_{b} r_{f}\right)-H_{0}^{(2)}\left(-j k_{b} r_{F}\right)\right]
$$

with $r_{F}=\sqrt{\left(x-x_{F}\right)^{2}+\left(y-y_{F}\right)^{2}}$.

Each wave function $\Psi_{b}$ should satisfy the homogeneous part of the dynamic plate bending equation (1). Vanmaele et al. [7] proposes the wave functions listed in 
Table 1. These wave functions are divided into two sets. For the first set of wave functions, the functions associated with the first wavenumber are cosine functions. For the second set, the functions associated with the first wavenumber are sine functions. It has been proven by Desmet [5] that the first set of wave functions is theoretically sufficient for the convergence of the WBM, provided that the domain is convex. In practice however, it is shown that at least a few functions of the second set are required for a beneficial convergence rate [9].

\begin{tabular}{|l|ll|}
\hline & Bending wave functions \\
\hline \multirow{3}{*}{ set 1} & $\Psi_{b_{1}}(\mathbf{x})=\cos \left(k_{b_{1}, x} x\right) \exp \left(-j k_{b_{1}, y} y\right)$ & $b_{1}=0,1, \cdots, n_{b_{1}}$ \\
& $\Psi_{b_{2}}(\mathbf{x})=\exp \left(-j k_{b_{2}, x} x\right) \cos \left(k_{b_{2}, y} y\right)$ & $b_{2}=0,1, \cdots, n_{b_{2}}$ \\
\hline \multirow{2}{*}{ set 2 } & $\Psi_{b_{1}}(\mathbf{x})=\sin \left(k_{b_{1}, x} x\right) \exp \left(-j k_{b_{1}, y} y\right)$ & $b_{1}=1, \cdots, n_{b_{1}}^{\prime}$ \\
& $\Psi_{b_{2}}(\mathbf{x})=\exp \left(-j k_{b_{2}, x} x\right) \sin \left(k_{b_{2}, y} y\right)$ & $b_{2}=1, \cdots, n_{b_{2}}^{\prime}$ \\
\hline
\end{tabular}

Table 1: Sets of wave functions for plate bending.

An infinite number of wave functions satisfy the homogeneous differential equations. A truncated set must be selected for each field variable. The selection is based on the dimensions $\left(L_{x} \times L_{y}\right)$ of the preferably smallest rectangular box circumscribing the problem domain. Desmet [5] chooses the first wavenumber such that an integer number of half wavelengths equals the dimension of the rectangular box in the corresponding direction,

$$
k_{x b_{1}}=\frac{b_{1} \pi}{L_{x}} \quad \text { and } \quad k_{y b_{2}}=\frac{b_{2} \pi}{L_{y}}
$$

with $\left(b_{1}, b_{2}\right)=0, \pm 1, \ldots$ The other component of the wavenumber is calculated from the structural bending wavenumber $k_{b}$ corresponding to the considered frequency,

$$
k_{y b_{1}}=\left\{\begin{array}{l} 
\pm \sqrt{k_{b}^{2}-k_{x b_{1}}^{2}} \\
\pm j \sqrt{k_{b}^{2}+k_{x b_{1}}^{2}}
\end{array} \text { and } \quad k_{x b_{2}}=\left\{\begin{array}{l} 
\pm \sqrt{k_{b}^{2}-k_{y b_{2}}^{2}} \\
\pm j \sqrt{k_{b}^{2}+k_{y b_{2}}^{2}}
\end{array}\right.\right.
$$

The number of bending wave functions $n_{b}$ that are included in the field variable expansion (6), is related to the frequency and the dimensions of the enclosing bounding box,

$$
n_{b}=4\left(n_{b_{1}}+1\right)+4\left(n_{b_{2}}+1\right)+4 n_{b_{1}}^{\prime}+4 n_{b_{2}}^{\prime},
$$


with,

$$
\frac{n_{b_{1}}}{L_{x}} \approx \frac{n_{b_{2}}}{L_{y}} \approx \frac{n_{b_{1}}^{\prime}}{L_{x}} \approx \frac{n_{b_{2}}^{\prime}}{L_{y}} \geq T \frac{k_{b}}{\pi}
$$

with $n_{b_{1}}, n_{b_{2}}, n_{b_{1}}^{\prime}$ and $n_{b_{1}}^{\prime}$ integer truncation values and with $T$ a user defined truncation parameter. In this way the largest wave number included in the model is $T$ times the structural wavenumber at this frequency. As a result, the number of wave functions increases approximately linearly with the excitation frequency.

\subsection{Evaluation of boundary conditions}

The wave functions proposed in Table 1 satisfy the governing partial differential equation (1). The boundary conditions are enforced through a weighted residual formulation. The residuals on the boundary conditions (4) are orthogonalized with respect to a weighting function $\tilde{w}_{z}$ or its derivative. In the case that all boundaries are clamped, the weighted residual formulation is expressed as,

$$
\int_{\Gamma} \mathcal{L}_{Q_{n}}\left[\tilde{w}_{z}\right] R_{w_{z}} \mathrm{~d} \Gamma+\int_{\Gamma} \mathcal{L}_{m_{n}}\left[\tilde{w}_{z}\right] R_{\theta_{z}} \mathrm{~d} \Gamma=0,
$$

with $\mathcal{L}_{Q_{n}}$ and $\mathcal{L}_{m_{n}}$ the differential operations for the generalised shear force and the bending moment defined as follows,

$$
\begin{aligned}
\mathcal{L}_{m_{n}} & =-D\left(\frac{\partial^{2}}{\partial n^{2}}+\nu \frac{\partial^{2}}{\partial s^{2}}\right) \\
\mathcal{L}_{Q_{n}} & =-D \frac{\partial}{\partial n}\left(\frac{\partial^{2}}{\partial n^{2}}+(2-\nu) \frac{\partial^{2}}{\partial s^{2}}\right) .
\end{aligned}
$$

Like in the Galerkin weighting procedure, used in the FEM, the weighting functions $\tilde{w}_{z}$ are expanded in terms of the same set of wave functions used in the field variable expansion (6):

$$
\tilde{w}_{z}=\sum_{b=1}^{n_{b}} \tilde{w}_{b} \Psi_{b}(\mathbf{x})=\mathbf{\Psi}(\mathbf{x}) \cdot \tilde{\mathbf{w}} .
$$

Substitution of the field variable expansion (6) and the weighting function expansion (15) into the weighted residual formulation (12) yields:

$$
\tilde{\mathbf{w}}^{T}[\mathbf{A} \cdot \mathbf{w}-\mathbf{f}]=0 .
$$

Since weighted residual formulation (12) should hold for any weighting function $\tilde{w}_{z}$, the expression between the square brackets in equation (16) must be zero. This yields a matrix equation consisting of $n_{b}$ algebraic equations in the $n_{b}$ unknown wave function contribution factors:

$$
[\mathbf{A}]\{\mathbf{w}\}=\{\mathbf{b}\}
$$


As for the Boundary Element Method (BEM) and in contrast with the FEM, the WBM yields a fully populated matrix, whose elements are complex and which cannot be decomposed into frequency independent submatrices. The big advantage of the WBM is, however, that the system matrices are substantially smaller in comparison with the element based techniques. This property, combined with the fast convergence of the WBM, makes it a less computationally demanding method for dynamic response calculations, which creates opportunities to tackle problems also in the midfrequency range.

\section{Introduction of a point mass}

In order to introduce a point mass into the WBM formulation, the particular solution of a point force (7) can be used. Newton's second law relates the force $F$ applied to the mass $m$ and its acceleration $a$,

$$
F=m a .
$$

This paper considers steady-state dynamics. Therefore, the former expression can be rewritten as:

$$
F=F_{m} e^{j \omega t}=m A e^{j \omega t}=-m \omega^{2} W_{m} e^{j \omega t},
$$

and thus,

$$
F_{m}=-m \omega^{2} W_{m}
$$

with $F_{m}, A$ and $W_{m}$ the amplitudes of respectively the force applied to the mass, the acceleration and the displacement of the mass, $\omega$ the circular frequency and $t$ the time.

The coupling of the point mass with the plate is achieved by imposing force equilibrium and displacement continuity between the point mass and the plate:

- According to Newton's third law, the law of reciprocal actions, the force exerted by the point mass on the plate is equal but directed opposite to the force exerted by the plate on the point mass. As a result, the expression of the particular solution of the inhomogeneous plate bending equation for a point force, equation (7), can be used to introduce the point mass inertia. The field variable expansion (6) can be rewritten as:

$$
w_{z}(\mathbf{x}) \approx \hat{w}_{z}(\mathbf{x})=\sum_{b=1}^{n_{b}} w_{b} \Psi_{b}(\mathbf{x})+\hat{w}_{F}(\mathbf{x})+\hat{w}_{m}(\mathbf{x}),
$$

with (using (7) and (20)),

$$
\hat{w}_{m}=-\frac{j m \omega^{2} W_{m}}{8 k_{b}^{2} D}\left[H_{0}^{(2)}\left(k_{b} r_{m}\right)-H_{0}^{(2)}\left(-j k_{b} r_{m}\right)\right]
$$


and

$$
r_{m}=\sqrt{\left(x-x_{m}\right)^{2}+\left(y-y_{m}\right)^{2}} .
$$

with $\mathbf{x}_{\mathbf{m}}\left(x_{m}, y_{m}\right)$ the position of the point mass. Introducing the expression (21) into the weighted residual formulation (12) with the same weighting functions (15), leads to the following matrix equation,

$$
\{\tilde{\mathbf{w}}\}^{T}\left[\left[\begin{array}{ll}
\mathbf{A} & \mathbf{A}^{\prime}
\end{array}\right]\left\{\begin{array}{c}
\mathbf{w} \\
W_{m}
\end{array}\right\}-\{\mathbf{f}\}\right]=0
$$

with,

$$
\begin{aligned}
\mathbf{A}^{\prime}= & -\int_{\Gamma} \mathcal{L}_{Q_{n}}\left(\Psi^{T}(\mathbf{x})\right) \frac{j m \omega^{2}}{8 k_{b}^{2} D}\left[H_{0}^{(2)}\left(k_{b} r_{m}\right)-H_{0}^{(2)}\left(-j k_{b} r_{m}\right)\right] \mathrm{d} \Gamma \\
& -\int_{\Gamma} \mathcal{L}_{m_{n}}\left(\boldsymbol{\Psi}^{T}(\mathbf{x})\right) \mathcal{L}_{\theta_{n}}\left(\frac{j m \omega^{2}}{8 k_{b}^{2} D}\left[H_{0}^{(2)}\left(k_{b} r_{m}\right)-H_{0}^{(2)}\left(-j k_{b} r_{m}\right)\right]\right) \mathrm{d} \Gamma .
\end{aligned}
$$

- Since the mass is connected rigidly to the plate, its displacement $W_{m}$ is equal to the evaluation of the field variable expansion (21) at the position of the mass, which, in its turn, can be expressed in function of the unknowns $w_{b}\left(b=1 \ldots n_{b}\right)$ and $w_{m}$,

$$
W_{m}=\sum_{b=1}^{n_{b}} w_{b} \Psi_{b}\left(\mathbf{x}_{\mathbf{m}}\right)+\hat{w}_{F}\left(\mathbf{x}_{\mathbf{m}}\right)+\hat{w}_{m}\left(\mathbf{x}_{\mathbf{m}}\right) .
$$

Adding the displacement continuity (26) to the system matrix, leads to the system matrix equation visualised in Figure 1. It should be noted that adding a point mass to the system does not influence the coefficients of the matrices without a point mass, it only results in an extra column and an extra row. This property will be used in Section 4.

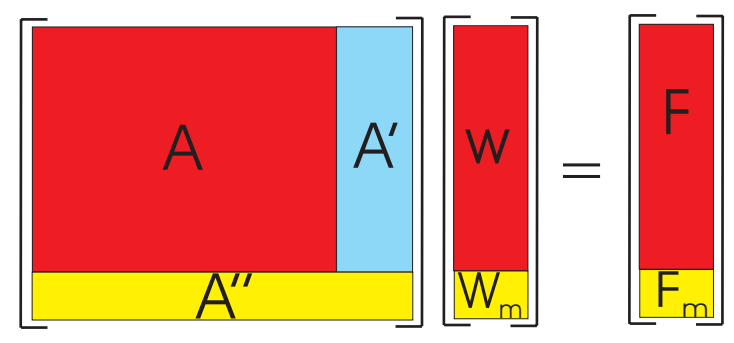

Figure 1: Visualisation of the system matrix equation.

Adding a point mass connected to the system with a spring and a damper can be dealt with very similarly. Only small modifications to the force equilibrium and displacement continuity equations are needed. 


\section{Use of WBM for efficient Monte Carlo simulations}

The previous section discusses the introduction of discrete point masses in the WBM. This section extends that approach to tackle problems with several point masses and proposes an efficient strategy to perform Monte Carlo simulations.

When the dynamic properties of a mechanical system exhibit a sufficient level of randomness, the response statistics become insensitive to the exact source of the variability [11]. Therefore, random point mass distributions can be used to introduce and represent a typical variability in a population of physical realisations of one nominal design.

When adding $n_{m}$ point masses to the system, the field variable expansion (21) becomes:

$$
w_{z}(\mathbf{x}) \approx \hat{w}_{z}(\mathbf{x})=\sum_{b=1}^{n_{b}} w_{b} \Psi_{b}(\mathbf{x})+\hat{w}_{F}(\mathbf{x})+\sum_{i=1}^{n_{m}} \hat{w}_{m_{i}}(\mathbf{x})
$$

Every point mass $m_{i}$ leads to an extra term in the field variable expansion and requires the introduction of an additional unknown, being the displacement amplitude of the point mass $W_{m_{i}}$. The additional terms in the field variable expansion lead, when used in the weighted residual formulation (12), to extra columns $\mathbf{A}^{\prime}$ in the system matrix without changing A, see Figure 1. For each extra unknown, so for each point mass, a continuity equation (26) is added $\left(\mathbf{A}^{\prime \prime}\right)$.

When performing a Monte Carlo simulation in order to determine the response statistics of a mechanical system, the response of $N$ realisations of the design needs to be calculated. When considering a system with a sufficiently high level of variability, each of these realisations can be constructed by randomly positioning $n_{m}$ point masses within the structure. Since the system building time takes up a large portion of the calculation time in a WBM calculation [9], the reuse of substeps in the model construction results in a significant gain in efficiency. As mentioned before, adding point masses does not change A. Therefore, for the $N$ samples, it is sufficient to only calculate this matrix once. Moreover, the weighting functions (15) should only be evaluated once along the boundaries. This approach leads to a considerable time reduction, as will be shown in the following section.

\section{Numerical example}

Figure 2 shows the considered problem geometry. It consists of a convex clamped aluminium plate, with a thickness of $0.002 \mathrm{~m}$, Young's modulus $E=70 \cdot 10^{9} \mathrm{~N} / \mathrm{m}^{2}$, Poisson coefficient $\nu=0.3$, damping loss factor $\eta=0.002$ and density $\rho=2790 \mathrm{~kg} / \mathrm{m}^{3}$. A unit point force is applied at $F(0.25 \mathrm{~m}, 0.125 \mathrm{~m})$. There are three response points, located at $w_{1}(0.25 \mathrm{~m}, 0.125 \mathrm{~m}), w_{2}(0.55 \mathrm{~m}, 0.31 \mathrm{~m})$ and $w_{3}(0.74 \mathrm{~m}, 0.16 \mathrm{~m})$.

In order to determine the response statistics of this system, a Monte Carlo analysis is performed. Each realisation consists of 15 randomly distributed masses, each 


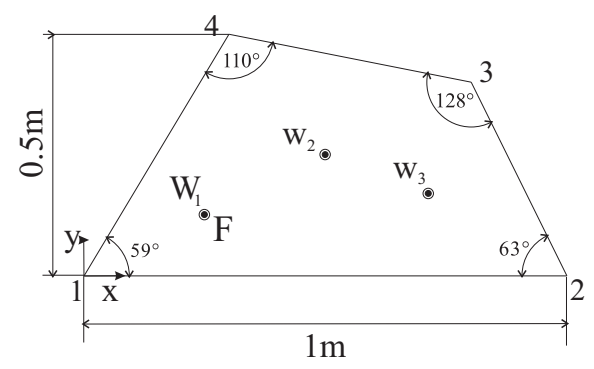

Figure 2: Problem geometry

weighing $15 \mathrm{~g}$, consituting in total approximately $15 \%$ of the plate mass.

In order to verify the implementation of the WBM with point masses, Figure 3 shows the frequency response functions of the displacement in point $w_{2}$ calculated with the WBM and the FEM with the same point mass distribution compared with a very fine FEM reference. The model parameters can be found in Table 5. The FE reference has 560598 dofs. For the WB calculation the number of degrees of freedom
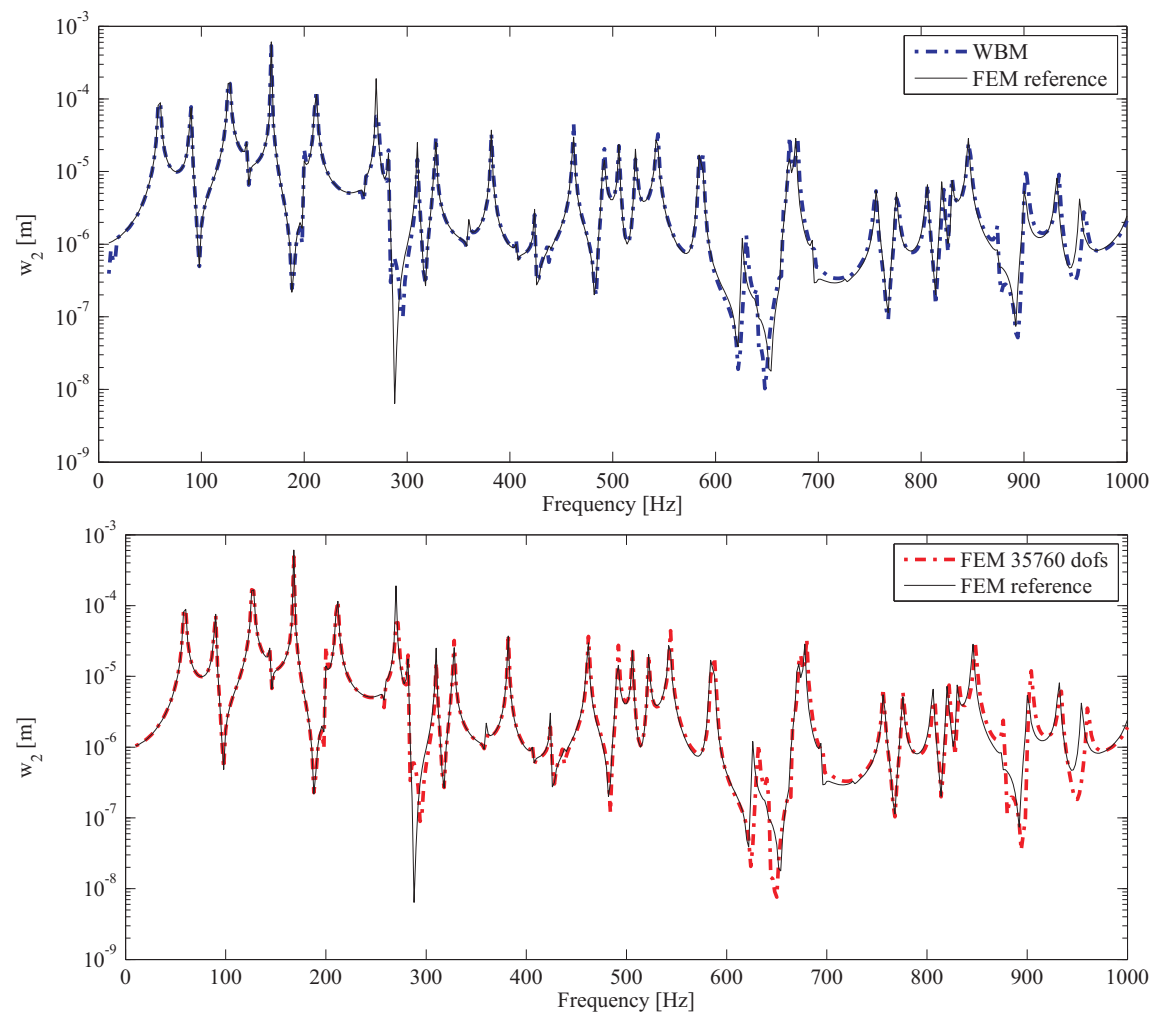

Figure 3: Comparison FEM and WBM with point masses

increases with frequency. A truncation factor $T=2$ is used. This results in 176 dofs at $1000 \mathrm{~Hz}$. The FE calculation has 35760 dofs. All FE-calculations are performed with MSC/Nastran. The WB calculation takes 334 seconds and the FE calculation takes 1036 seconds for 496 frequency lines, on a $3 \mathrm{GHz}$ Intel Pentium 4 running a 


\begin{tabular}{|l|c|c|c|}
\hline Model & Dofs & \#Elements $/ \lambda$ & CPU time \\
\hline WBM & 176 at $1000 \mathrm{~Hz}$ & - & 334 secs \\
FEM & 35760 & 14 & 1036 secs \\
FE reference & 560598 & 55 & \\
\hline
\end{tabular}

Table 2: Model parameters of the WB, the FE and the FE reference model.

Linux-operating system. Although both have a similar accuracy, the WBM is 3 times faster. It should be noted that the FEM calculation time only includes the solving time, the WBM calculation time includes the whole process (building, solving and postprocessing).

Figure 4(a) shows the average calculation time for the whole frequency range per sample for a Monte Carlo simulation with the same system as above, including 15 discrete point masses of $15 \mathrm{~g}$ randomly distributed, for an increasing amount of samples with the WBM adopting the system submatrix reuse. The average time decreases rapidly between 1 and 30 samples and stagnates at a level of about $45 \%$ of the time for the calculation of 1 sample. This stagnation is caused by the fact that the building of the common part divided by the number of samples is becoming small compared to the evaluation of the particular solutions for the point masses and the system solving time. However, when going up to higher frequencies or larger systems the gain will be larger because the degrees of freedom and thus the common part of the system will become larger, while the number of point mass unknowns remains the same. It should be noted that the average time for a FEM Monte Carlo is 1036 seconds and is constant in function of the number of samples. The evolution of the total time in function of the number of samples is shown in Figure 4(b) for the FEM, the WBM without the reuse of the common part and the WBM with the reuse of system submatrices. The WBM with system submatrix reuse gives a clear advantage.

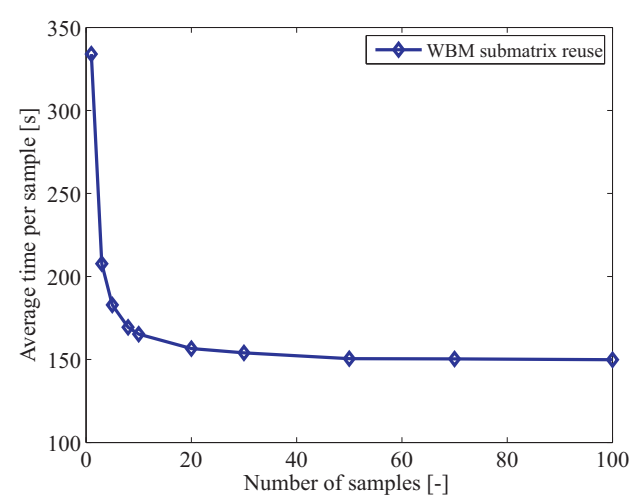

(a) Average time (WBM matrix reuse)

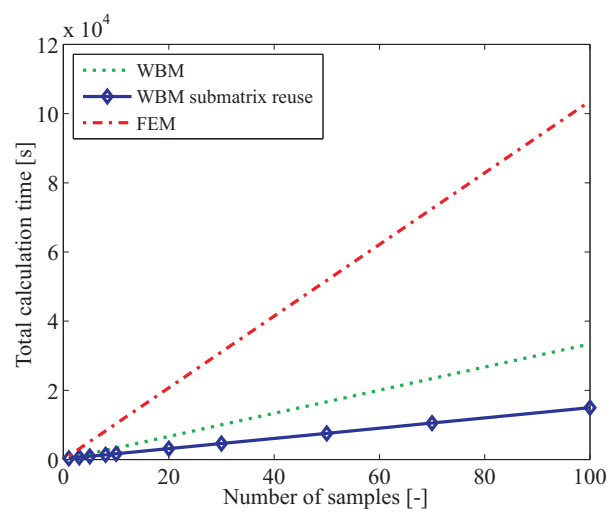

(b) Total time

Figure 4: Evolution of average time per sample in function of number of samples for the WBM with system submatrix-reuse (left) and total time (right).

Figure 5(a) compares the average of the predictions of 100 samples averaged in 
the three postprocessing points calculated with the WBM and with the FEM and Figure 5(b) shows the outer bounds. It can be seen that the influence of the point masses increases with frequency up to $300-400 \mathrm{~Hz}$ because the spread on the results increases. At $300-400 \mathrm{~Hz}$ the "stochastic" zone has been entered. The results of the FE and the WB calculations generally agree, although starting from $300 \mathrm{~Hz}$ the small peaks do not coincide exactly because the FEM suffers from some dispersion. The WB with submatrix reuse calculation takes 14981 seconds and the FE calculation 103600 seconds.

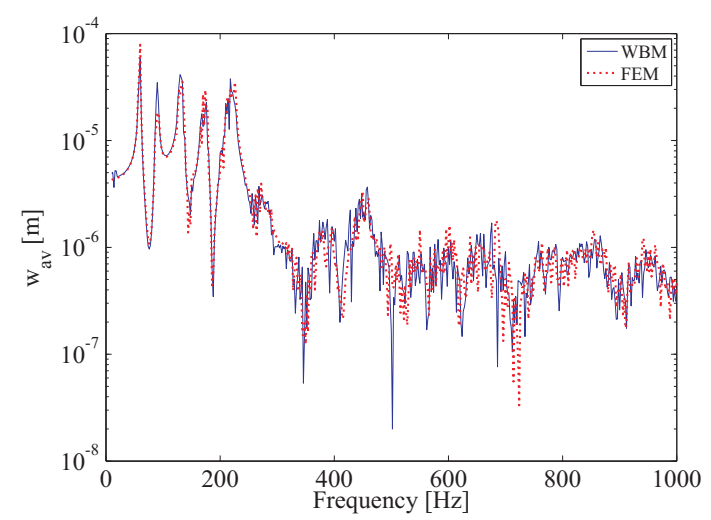

(a) Averaged response

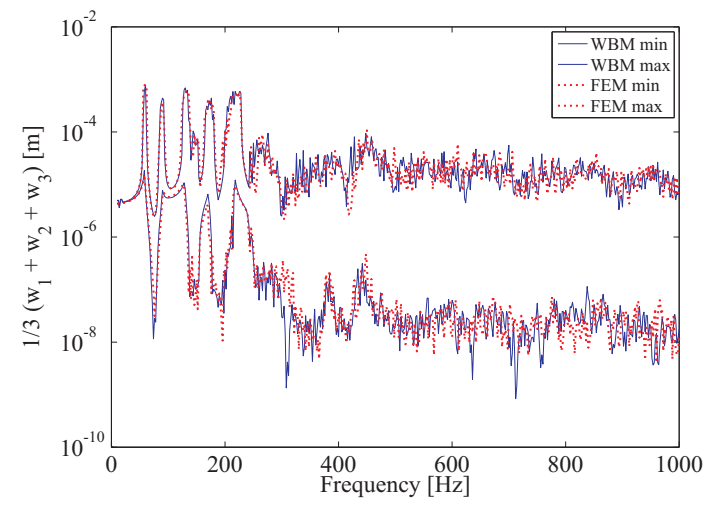

(b) Outer bounds

Figure 5: Response averaged over 100 samples and the outer bounds for FEM and WBM.

\section{Conclusions and future work}

This paper reports on the developments of the WBM for the steady-state dynamic analysis of the bending behaviour of flat plates with point masses. The WBM is a novel numerical deterministic prediction technique, based on an indirect Trefftz approach.

The paper starts with the description of the WBM for plate bending. Once this has been defined, it is possible to describe the addition of point masses to the system. In this paper, an approach is used which introduces the effect of each point mass using a particular solution, based on the displacement field resulting from a point force applied on an infinite plate. Each point mass leads to an additional unknown, but also to a displacement continuity equation. The extension to point masses connected to the plate with springs and dampers is straightforward and can be used in the near future.

An efficient Monte Carlo simulation with random point mass distributions is proposed. This efficiency improvement is achieved by reusing parts of the system matrix, and therefore saving on building time.

The last section gives a numerical example, which demonstrates the efficiency of the proposed method compared to the FEM. As a result, it is possible to tackle the stochastic range with the deterministic WBM within reasonable calculation times. 
An interesting and logic next step in this research is the development of this WBM with reusing system submatrices for larger systems, not necessarily consisting of only flat plates. These larger systems may exhibit partially a deterministic, partially a probabilistic behaviour. Aside from this, it would be interesting to adopt the WBM in the hybrid deterministic-probabilistic framework proposed by Shorter and Langley [20]. In this way, the computationally expensive Monte Carlo simulations can be avoided.

\section{Acknowledgements}

Karel Vergote and Bert Van Genechten are Doctoral Fellows of the Fund for Scientific Research - Flanders (F.W.O.), Belgium.

\section{References}

[1] O.C. Zienkiewicz, R.L. Taylor, J.Z. Zhu, P. Nithiarasu, The Finite Element Method - The three volume set (6th. ed.), Butterworth-Heinemann, 2005.

[2] C.A. Brebbia, J. C. F. Telles, and L. C. Wrobel, "Boundary Element techniques: theory and applications in engineering”, Springer Berlin, 1984.

[3] J. Dominguez, "Boundary Elements in Dynamics", Computational Mechanics Publications, Southampton, 1993.

[4] R.H. Lyon, R.G. DeJong, "Theory and application of statistical energy analysis (2nd ed.)", Butterworth-Heinemann, 1995.

[5] W. Desmet, "A Wave based prediction technique for coupled vibro-acoustic analysis." Ph.D. dissertation, Katholieke Universiteit Leuven, Departement Werktuigkunde, Leuven, 1998 (http://www.mech.kuleuven.be/mod/wbm/phd_dissertations).

[6] E. Trefftz, "Ein Gegenstck zum Ritzschen Verfahren", in "Proceedings of the Second International Congress on Applied Mechanics",Zurich, Switzerland, 131-137, 1926.

[7] C. Vanmaele, D. Vandepitte, W. Desmet, "An efficient wave based prediction technique for plate bending vibrations”, Comput. Methods Appl. Mech. Engrg., 196, 3178-3189, 2007.

[8] C. Vanmaele, D. Vandepitte, W. Desmet, An efficient wave based prediction technique for dynamic plate bending problems with corner stress singularities, Accepted for publication in Comput. Methods Appl. Mech. Engrg.,2007.

[9] C. Vanmaele, "Development of a wave based prediction technique for the efficient analysis of low- and mid-frequency structural vibrations." Ph.D. dissertation, Katholieke Universiteit Leuven, Departement Werktuigkunde, Leuven, 2007 (http://www.mech.kuleuven.be/mod/wbm/phd_dissertations).

[10] C. Vanmaele, K. Vergote, D. Vandepitte, W. Desmet, An efficient wave based prediction technique for the dyanamic analysis of 3D plate assemblies, Submitted for publication in Comput. Methods Apll. Mech. Engrg., 2008. 
[11] R. S. Langley, V. Cotoni, "Response variance prediction in the statistical energy analysis of built-up systems”, J. Acoust. Soc. Am., 115(2), 706-718, 2004.

[12] K. Binder, "Application of the Monte Carlo Method in Statistical Physics", Springer-Verlag, Berlin, 1984.

[13] R.Y. Rubenstein, "Simulation and the Monte Carlo Method", Wiley, New-York, 1981.

[14] A. Leissa, "Vibration of plates", Acoustical Society of America, Woodbury, New-York, 1993.

[15] R.D. Mindlin, "Influence of rotary inertia and shear on flexural motions of isotropic, elastic plates”, J. Appl. Mech. ASME, 18, 31-38, 1951.

[16] E. Reissner, "The effect of transverse shear deformation on the bending of elastic plates”, J. Appl. Mech. ASME, 12, A69-A77, 1945.

[17] L. Cremer, M. Heckl, E.E. Ungar, "Structure-borne Sound: structural vibrations and sound radiation at audio frequencies”, Springer-Verlag, Berlin, 1973.

[18] C.S. Huang, O.G. McGee, A.W. Leissa, "Exact analytical solutions for free vibrations of thick sectorial plates with simply supported radial edges”, International Journal of Solids and Structures, 31, 1609-1631, 1994.

[19] M. Junger, D. Feit, “Sound, Structures, and Their Interactions”, Acoustical Society of America, 1993.

[20] P.J. Shorter, R.S. Langley, “Vibro-acoustic analysis of complex systems”, Journal of Sound and Vibration, 288, 669-699, 2005. 